\title{
Experiment Regarding Magnetic Fields with Gravity
}

\section{Short Title: Gravity within Electricity Series}

\author{
Jong-hoon Lee*
}

Science \& Research Center, Seoul National University College of Medicine; Seoul, South Korea.

*Corresponding author. Email: science@ research.re.kr

\begin{abstract}
When gravity exists in magnetic fields, gravity interacts with these magnetic fields to generate electricity in the direction the Earth spins or in the opposite direction. The relationships between electricity, voltage, and time can be redefined by analyzing data for 0.1 seconds. The voltage can be recorded on the x-axis, and time can be recorded on the y-axis. The electric potential energy generated due to interactions between gravity and magnetic seas can explain the time-dependent or time-independent equations of the Schrödinger equation through the data analysis.
\end{abstract}

One-Sentence Summary The Earth's gravity on the mesoscopic scale generates electricity in the magnetic sea. 


\section{Main Text}

A magnetic/electric field can vary the gravitational field. The observed gravitational redshift/blueshift, the bending of light by gravity from the numerical resolution of the coupled Einstein-Maxwell equations in cylindrical symmetry ${ }^{1}$, and the Shapiro time delay showed that gravity performs actions on photons ${ }^{2}$. We know that a photon varies according to the gravitational field's energy from the energy conservation law. According to the law of conservation of energy describing the gravitational redshift and quantum field theory, some researchers obtained equations for the interactions between gravitational and magnetic/electric fields ${ }^{3}$. Detection of astrophysical gravitational waves with laser interferometers such as LIGO can explore relatively low-frequency ranges (typically less than $1-10 \mathrm{kHz}$ ). Electromagnetic detectors of gravitational waves could explore a higher frequency range, typically from $\mathrm{kHz}$ to $100 \mathrm{GHz}$ when using radio-frequencies or from $100 \mathrm{GHz}$ to $\mathrm{THz}$ when using microwaves.

It is tough and almost impossible to make electromagnetic detectors of gravitational waves ${ }^{4}$. It would be better to make electromagnetic detectors of gravitational collisions within a more prosperous Earth's gravitational field. Gravitation is fragile interactions compared to electromagnetism and nuclear forces. Generating gravitational fields requires storing large amounts of energy on a short scale. Nonetheless, controlling gravity, for instance, through producing then detecting artificially generated gravitational collisions, does not require any new physics nor technology ${ }^{5}$. We tried to demonstrate that gravity induces magnetic seas by monitoring the electricity, voltage, and time in electric wire.

\section{Results}


We connected the gravity generator (GG) to a TDS 2014 four-channel digital storage oscilloscope (100 MHz, $1 \mathrm{GS} / \mathrm{s}$ ) (Supplemental Sections 5 and 6). Furthermore, the voltage was measured during experiments D1 to F6 in order while the generator was stationary through the monitor. Gravity only generates electric potentials (Supplemental Section 6).

The data were $3.60 \rightarrow 3.20 \rightarrow 3.40 \rightarrow 3.20 \rightarrow 4.00 \rightarrow 3.60 \rightarrow 3.40 \rightarrow 3.80 \rightarrow 4.00 \rightarrow 5.00 \rightarrow 3.40 \rightarrow 2.80 \rightarrow 3.80 \rightarrow 4.00 \rightarrow 3.40 \rightarrow 4.00$ $\rightarrow 3.60 \rightarrow 3.40 \rightarrow 4.00 \rightarrow 3.80 \rightarrow 3.40 \rightarrow 4.20 \rightarrow 3.40 \rightarrow 3.80 \rightarrow 3.40 \rightarrow 3.20 \rightarrow 3.40 \rightarrow 4.00 \rightarrow 3.20 \rightarrow 4.20 \rightarrow 3.40 \rightarrow 3.60 \rightarrow 3.00 \rightarrow 3.20 \rightarrow$ $3.80 \rightarrow 4.20 \rightarrow 3.40 \rightarrow 3.60 \rightarrow 3.40 \rightarrow 4.00 \rightarrow 3.40 \rightarrow 4.00 \rightarrow 3.80 \rightarrow 3.00 \rightarrow 3.80 \rightarrow 4.00 \rightarrow 3.00 \rightarrow 3.20 \rightarrow 3.80 \rightarrow 3.20 \rightarrow 3.40 \rightarrow 3.80 \rightarrow 4.0$ $0 \rightarrow 3.40 \rightarrow 4.40 \rightarrow 3.20 \rightarrow 4.00 \rightarrow 7.60 \rightarrow 4.60 \rightarrow 3.60 \rightarrow 4.00 \rightarrow 3.80(\mathrm{~V})$ while the generator was stationary.

The GG was connected to measure the amount of electricity. When it rotated faster, more electricity was produced. We measured electricity three times (A, B, C) while the GG rotated (Supplemental Section 3 - Table 1) and three times when the GG was stationary (Supplemental Section 4 -Table 2). The electricity generated in a stationary state was examined more precisely than that in a rotated state: Experiments D1, E1, F1, D6, E6, and F6 (Table 1). predicts space and time's frothiness. Nevertheless, gravity (gravitons or gravitational waves) was the sole factor influencing this experiment's magnetic fields in a firmly fixed Hieut space $(\mathrm{H}$ : Hieut) on Earth (Fig. 1).

The data of experiments D1, E1, and F1 from the stationary GG were analyzed in rows 312 to 412 to remove the effects of weight, the time interval was 0.1 seconds, and there were 100 measured points ${ }^{6}$. The $\mathrm{x}$-axis represents time, and the $\mathrm{y}$-axis denotes voltage and current in a radial chart. Gravity and magnetic force were in balanced states while the GG was stopped, but in experiment D1, the voltage ranged from -11.1 to 13.0 microvolts, and the current ranged from -1.90 to 29.0 microamps. In experiment E1, the voltage ranged from -11.9 to 9.60 microvolts, and the current ranged from -2.00 to 2.40 microamps. In experiment F1, the voltage ranged from -8.10 to 14.1 microvolts, and the current ranged from -3.30 to 3.00 microamps (Fig. 2). 
The amounts of electricity generated in the vacuum chamber were measured again (Supplemental Section 7). This vacuum experiment demonstrated the changes in the amount of current generated as the barometric pressure decreased. It indicated that gravity could be transferred to electricity using air as a medium. If electricity is generated in a vacuum, this signifies the presence of fluids or particles without air.

We measured the currents in order from 1 to 10000 and summed them all. The changes in the magnitude of the current were observed as the barometric pressure decreased.

The 4th measurement, measured for 10 seconds in the vacuum chamber experiment, was selected for data analysis: the data were analyzed from point 4000 to point 4999 , with this interval corresponding to 1 second. The $\mathrm{x}$-axis represented time, and the $\mathrm{y}$-axis represented voltage. Then, the $\mathrm{x}$-axis represented voltage, and the $\mathrm{y}$-axis represented time. There were 107 cases where the voltage had two times and 16 cases where voltage had three times, and 1 case where voltage had four times. We could find cases containing the same voltage but at different times (Fig. 3).

The data were analyzed from point 4000 to point 4999, with this interval corresponding to 1 second from the 4 th measurement measured in the vacuum chamber. It includes the cases where the voltage remains the same while the time is different. There are 107 cases of one voltage/ two times and 16 cases of one voltage/ three times, and 1 case of one voltage/ four times (Supplemental Section 7. DATA).

\section{Discussion}

It was not until 1989 that microampere measurements were possible. Kibble Balance measures mass by supplying current, but in this experiment, only gravitational mass was supplied. 


\section{On the Possible Relationship between Gravity and Electricity}

The GG produced a certain amount of electricity in the stationary state. The current was produced in the downward direction when the bearing cover was pressed. Furthermore, electricity was also produced upward when the bearing cover jumped near the magnetic field. Electricity was generated when dumbbells 1 and 2 were placed on the GG rotating ${ }^{7}$ or stationary

${ }^{8}$. (Supplemental Sections 3 and 4)

However, electricity cannot be generated according to Maxwell's equation when the GG is stationary in the equilibrium state.

[Maxwell's equation]

$$
\frac{\partial B x}{\partial x}+\frac{\partial B y}{\partial y}+\frac{\partial B z}{\partial z}=0 \quad \varepsilon^{\mu v \kappa \lambda} \partial_{v} F_{\kappa \lambda}=0
$$

If the current and voltage were generated at the equilibrium state, there is no other way to explain this phenomenon other than describing it as the generation of electricity by dividing particles according to the Weinberg-Witten theorem ${ }^{9}$. The LIGO and Virgo detectors observed that gravitational waves travel simultaneously as light and not more slowly. As Einstein's general theory of relativity had predicted, those observations confirmed the Lorentz invariance principle $10-12$

We can express the magnetic-electric field through its respective gravitational analogues using the proportionality coefficient $\mathrm{k}$. This coefficient $\mathrm{k}$ depends on the ratio of mass and charge. Although the last coefficient is minimal, it represents the ratio is between the electromagnetic and gravitomagnetic permittivity and permeability ${ }^{13}$.

(Example: Coupled Maxwell-Einstein equations in a weak field approximation) ${ }^{13}$ 
$\operatorname{rot} \vec{E}=-\frac{1}{\kappa} \frac{\partial \vec{B}_{g}}{\partial t}$

$\operatorname{rot} \vec{B}=\frac{e}{m} \mu_{0} \rho_{m} \vec{v}+\frac{1}{\kappa} \frac{1}{c^{2}} \frac{\partial \vec{g}}{\partial t}$

(Gravitation ->Electromagnetism in the weak field approximation)

(Coupled Maxwell-Einstein equations in a weak field approximation) ${ }^{13}$

$$
\begin{aligned}
& \operatorname{rot} \vec{g}=-\kappa \frac{\partial \vec{B}}{\partial t} \\
& \operatorname{rot} \vec{B}_{g}=-\frac{m}{e} \mu_{g} \rho \vec{v}-\kappa \frac{1}{c^{2}} \frac{\partial \vec{E}}{\partial t}
\end{aligned}
$$

(Electromagnetism->Gravitation in the weak field approximation)

It was found that every EM field is coupled to a gravitoelectric and gravitomagnetic field

13. If gravity is present in the EM field, gravity interacts with it to generate electricity due to magnetic field changes.

\section{Time to the Voltage by the Dirac Equation}

Experiments on the electrical conversion of gravity were used to obtain the data. The data were reconstructed with the voltage on the $\mathrm{x}$-axis and the time on the y-axis. The D1, E1, and F1 experiments were measured while the GG was stationary (Supplemental Sections 2 and 4).

The time-independent equations of the Schrödinger equation represent the potential (voltage) formed between an electron and the nucleus. Voltage is formed among the nucleus and the electron clouds because their distances are not relatively long. The differences in time indicate the differences in voltage. The D1, E1 and F1 voltage-time graphs were rearranged in order of size based on the obtained voltages (Fig. 2). The relationships between time and voltage 
explain the time-dependent Schrödinger equation and the time-independent Schrödinger equation. The super-many-time theory showed that each infinite term could be regarded as a correction between the mass and charge of an electron ${ }^{14-16}$. The Schwinger model exhibits spontaneous symmetry breaking due to a chiral condensate derived from a pool of instantons. This model can $5 \quad$ be solved precisely ${ }^{17-19}$.

The Dirac equation, which is satisfied when a particle with a stationary mass of $\mathrm{m}$ moves freely without being influenced by the outside environment, is expressed as follows ${ }^{20}$ :

$$
i \hbar \sum_{\mu=0}^{3} \gamma^{\mu} \frac{\partial}{\partial x^{\mu}} \psi-m c \psi=0
$$

The theory of self-similar geometric structures is used to treat infinities arising in calculated quantities by altering these quantities' values to compensate for the effects of their self-interactions, such as the magnetic moment of an electron ${ }^{21,22}$.

According to this experiment, each voltage point has its own specific time(s). This gravity-electricity experiment might define the relationship between time(s) and voltage as the new renormalization theory. 


\section{References and Notes}

1 Füzfa, A. How current loops and solenoids curve spacetime. Physical Review D 93, 024014, doi:10.1103/PhysRevD.93.024014 (2016).

2 Pound, R. V. \& Rebka, G. A. Gravitational Red-Shift in Nuclear Resonance. Phys. Rev. Lett. 3, 439-441, doi:10.1103/PhysRevLett.3.439 (1959).

3 Zhu, Y. Gravitational-magnetic-electric field interaction. Results Phys. 10, 794-798, doi:10.1016/j.rinp.2018.07.029 (2018).

4 Fuzfa, A. Electromagnetic Devices for the Directional Emission and Reception of Gravitational Waves. (2017). Füzfa, A. Electromagnetic Gravitational Waves Antennas for Directional Emission and Reception. arXiv preprint arXiv:1702.06052 (2017).

6 Lee, J. H. The Data of Experiment D1, E1, F1. (Center for Open Science, 2020).

7 Lee, J. H. Audiovisual Material of Section S3. Experiments $(A, B, C)$. (YOUTUBE, 2020). Lee, J.

9 Weinberg, S. \& Witten, E. Limits on massless particles. Phys. Lett. B 96, 59-62 (1980).

10 Abbott, B. P. et al. GW170817: observation of gravitational waves from a binary neutron star inspiral. Phys. Rev. Lett. 119, 161101, doi:10.1103/PhysRevLett.119.161101 (2017).

11 Margutti, R. et al. The electromagnetic counterpart of the binary neutron star merger LIGO/Virgo GW170817. V. Rising X-ray emission from an off-axis jet. Astrophys. J. 848, L20, doi:10.3847/2041-8213/aa9057 (2017).

12 Cho, A. A spacetime tremor and a celestial light show. Science 358, 282-283, doi:10.1126/science.358.6361.282 (2017).

13 Tajmar, M. \& De Matos, C. Coupling of electromagnetism and gravitation in the weak field approximation. J. Theor. 3, arXiv (2001).

14 Kroll, N. M. \& Lamb Jr, W. E. On the self-energy of a bound electron. Phys. Rev. 75, 388 (1949).

15 Fukuda, H., Miyamoto, Y. \& Tomonaga, S. I. A self-consistent subtraction method in the quantum field theory. II-1. Prog. Theor. Phys. 4, 47-59, doi:10.1143/ptp.4.47 (1950).

16 Schweber, S. S. QED and the men who made it: Dyson, Feynman, Schwinger, and Tomonaga. (Princeton University Press, 1994).

17 Schwinger, J. The theory of quantized fields. I. Phys. Rev. 82, 914 (1951).

18 Schwinger, J. The theory of quantized fields. II. Phys. Rev. 91, 713 (1953).

3519 Schwinger, J. Gauge invariance and mass. II. Phys. Rev. 128, 2425 (1962).

20 Dirac, P. A. M. The Principles of Quantum Mechanics. Vol. 27 (Clarendon Press, 1981).

21 Zinn-Justin, J. Quantum field theory and critical phenomena. (Clarendon Press, 1996).

22 Kusch, P. \& Foley, H. M. The magnetic moment of the electron. Phys. Rev. 74, 250-263, doi:10.1103/PhysRev.74.250 (1948).

Lee, J. H. Gravity Generator-Voltage (0) and current (0) in Equilibrium State on Experiment F4 6380 (6379). OSF, Editor. 7 Nov. 2020. (Center for Open Science, 2020). 
Acknowledgments: Il-whan Kim designed the generator. He is a generator developer with 40 years of experience and asserted that electricity could never be generated from this device; James

Oh, general manager of Jays, Inc., in South Korea, compiled the data to assist with the Keysight B2901A electricity experiment when running the generator. Taeil Chung of the Extreme Engineering Research Center at the Korea Institute of Civil Engineering and Building Technology operated a vacuum chamber to assist with this experiment. Woo-Seung Maeng and Jae-yeol Shim provided the necessary expenses for this paper. D. Młodziankowski provided the theory of gravitational experiments so that the experiments could be explained according to the logic flow.

Funding

No funding

Author Contributions: JH Lee developed the theory of gravity generation, performed the experiments and wrote the manuscript.

\section{Competing Interests}

Authors declare that they have no competing interests.

\section{Data and Materials Availability}

All data is published by OSF: Gravity to Electricity as Quantum (https://osf.io/ntuda/).

\section{Supplementary Materials}

\section{Supplementary Text}

Supplemental Section 1. Gravity generator design

Supplemental Section 2. Keysight B2901A manual

Supplemental Section 3. Experiments (A, B, C - Table S1)

Supplemental Section 4. Experiments (D, E, F - Table S2)

Supplemental Section 5. Experiments (Voltage only while rotating) 
Supplemental Section 7. Experiments (Current, voltage and time while stationary in the vacuum chamber)

\section{Figs. S1 to S6}

Figure 1. Composition for a balanced arrangement of gravity and magnetic field

Figure 2. A schematic diagram of gravity that navigates in magnetic sea

Figure 3. Gravity Generator blueprint

Figure 4. Gravity Generator parts and test operation

Figure 5. B2900A Series Manual

Figure 6. Gravity Generator in the Vacuum Chamber

\section{Tables S1 to S6}

Table S1. Electricity measured while the generator rotating

Table S1-1 Electricity data rearranged while the generator rotating

Table S2. Electricity measured while the generator stationary

Table S2-1. Electricity data rearranged while the generator stationary

Table S3. Voltage measurement data while Rotating

Table S4. Voltage measurement data while Stationary

Table S5. Voltage measurement in the vacuum chamber

Table S6. Time and voltage measurement in the vacuum chamber

\section{Captions for Movies S1 to S3}

Movies S1. 2019.10.04 Discovery of Gravity to Electricity on Gravity Generator Movies S2. 2019.10.10 Voltage measurement data while Rotating on Gravity Generator Movies S3. 2019.10.10 Voltage measurement data while stationary on Gravity Generator

\section{Captions for Data S1 to S3}

Data S1 2019.10.04. Preparations before experimenting with the air and first simulations.

25 Data S2 2020.06.18. Experiments with the gravity generator in the vacuum chamber.

Data S3 2020.06.18. Vacuum chamber \& gravity generator in a vacuum. 
Figures

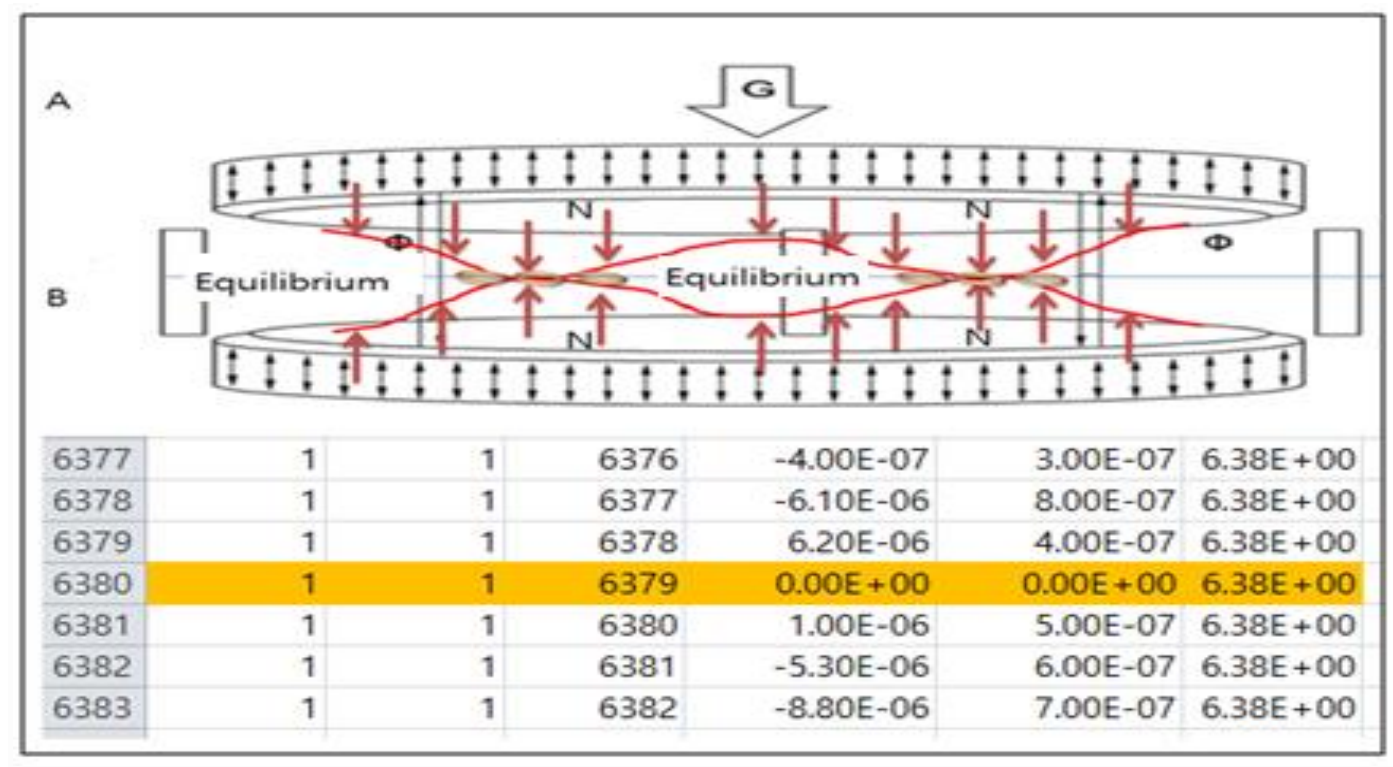

Fig. 1. Gravity generator - 0 voltage and 0 current in the equilibrium state during experiment F4 6380 (6379): in the direction Earth spins under gravity and in the opposite 5 direction under the repulsive magnetic force ${ }^{23}$. 

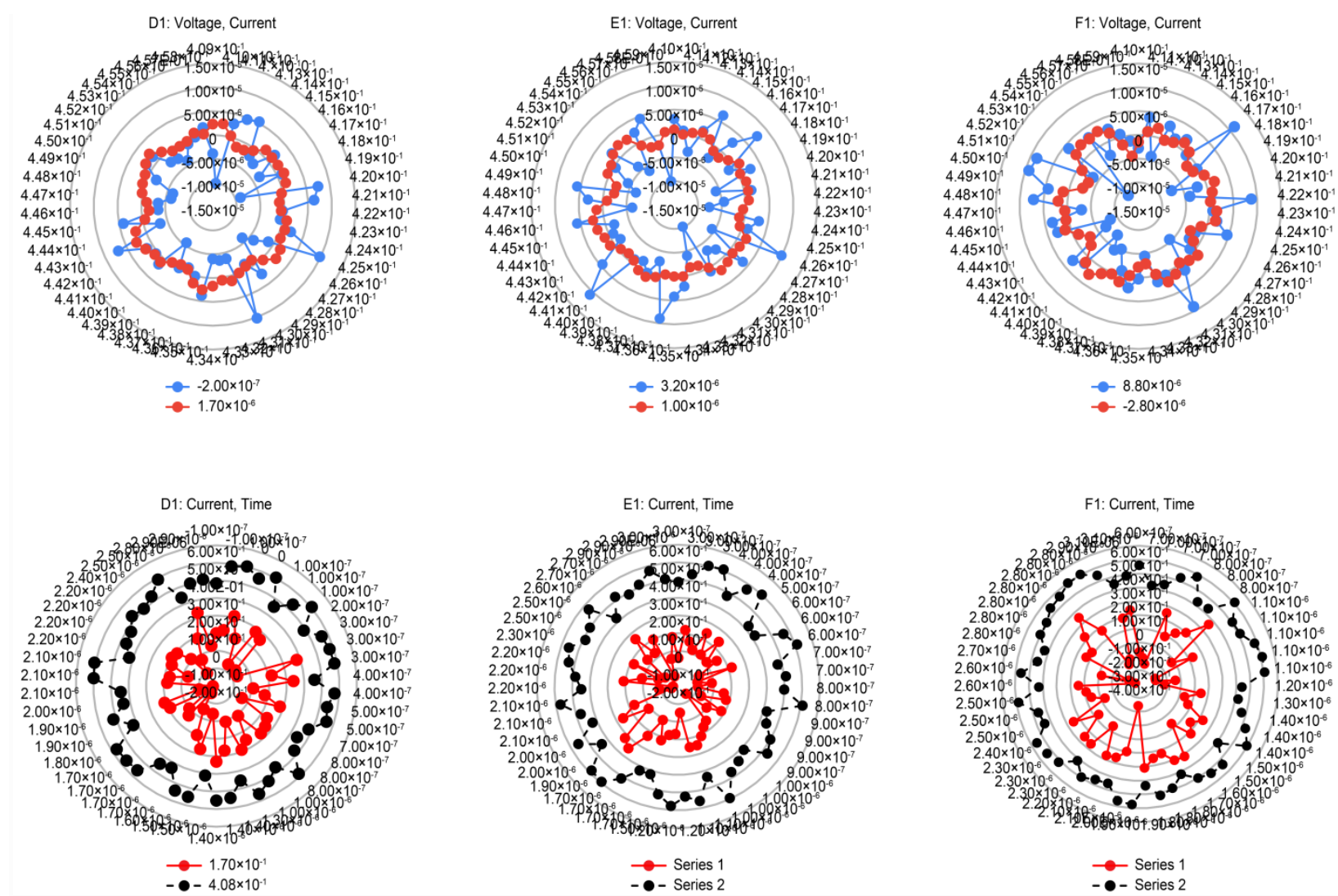

Fig. 2. First row: Experiments D1, E1 and F1 were measured when the gravity generator stopped. This includes the data from points 408 of D1 (409 of E1, F1)) to 458 of D1 (459 of E1, F1) of the raw data ${ }^{6}$. The blue is

the voltage, and the red is the current. Located on the y-axis, the round black x-axis is the time in 0.001-second increments. Second row: D1, E1 and F1 voltage-current-time graphs. These include the data from columns C100:E150 of the Excel file. The $\mathrm{x}$-axis is the voltage. The black is the time, and the red is the current. The time and current are located on the y-axis. The current was displayed on the graph by multiplying each current by $10^{6}$. 

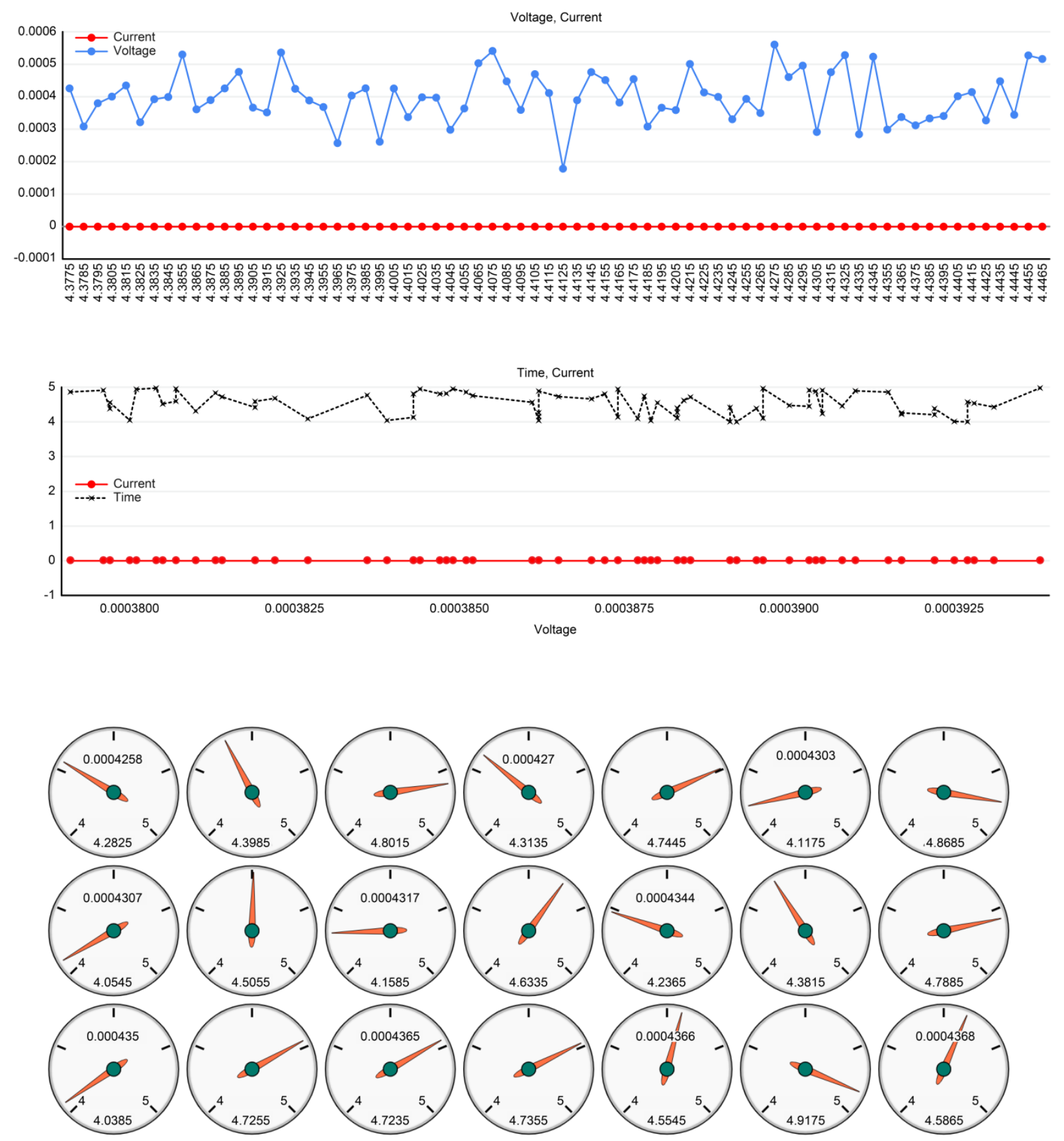

Fig. 3. Graph with the same voltage but different times. 
Tables

Table 1. The electricity generated while the GG was rotating and stationary was measured on October $10,2019$.

\begin{tabular}{|c|c|c|c|c|c|c|}
\hline & \multicolumn{3}{|c|}{ CH1 Current (A) } & \multicolumn{2}{|c|}{ CH1 Voltage (V) } & \multirow{2}{*}{$\begin{array}{l}10 \text { second }-100,000 \\
\text { point (every } 0.001 \\
\text { s) }\end{array}$} \\
\hline & Maximum & Minimum & Sum & Maximum & Minimum & \\
\hline $\mathrm{A} 2$ & 0.0007458 & -0.0016802 & -0.1374131 & 0.0000179 & -0.0000208 & -0.0002372 \\
\hline A3 & 0.0010683 & -0.0017568 & -0.1140228 & 0.0000251 & -0.000031 & 0.0017442 \\
\hline A4 & 0.0026882 & -0.0011917 & 0.1360524 & 0.0000189 & -0.0000229 & -0.0002788 \\
\hline A6 & 0.0004334 & -0.0003605 & 0.0101899 & 0.0000194 & -0.0000186 & -0.0022982 \\
\hline B1 & 0.0005052 & -0.0003646 & 0.0056757 & 0.0000209 & -0.0000189 & 0.0005494 \\
\hline B2 & 0.0008837 & -0.0021383 & -0.1239456 & 0.0000237 & -0.0000212 & 0.0019692 \\
\hline B3 & 0.0008959 & -0.0032941 & -0.1080884 & 0.0000406 & -0.0000417 & -0.0024536 \\
\hline B4 & 0.0024636 & -0.0011921 & 0.0723971 & 0.000019 & -0.0000214 & -0.0013109 \\
\hline B5 & $\begin{array}{l}\text { The test recorder } \\
\text { overwrote the } \\
\text { measurement data } \\
\text { the notebook. }\end{array}$ & & & & & \\
\hline B6 & 0.0004619 & -0.0003687 & -0.0004063 & 0.0000211 & -0.0000182 & 0.0013422 \\
\hline $\mathrm{C} 1$ & 0.0004593 & -0.0003969 & 0.033351 & 0.0000198 & -0.0000202 & -0.0034343 \\
\hline $\mathrm{C} 2$ & 0.0004714 & -0.0012035 & -0.0831234 & 0.0000191 & -0.0000231 & -0.0006971 \\
\hline $\mathrm{C} 3$ & 0.0026662 & -0.0019067 & -0.0950192 & 0.0000236 & -0.0000218 & 0.0001664 \\
\hline $\mathrm{C} 4$ & 0.0029132 & -0.0016932 & 0.118348 & 0.0000194 & -0.0000221 & -0.0022711 \\
\hline $\mathrm{C} 5$ & 0.0052364 & -0.0065765 & 0.1328696 & 0.0000721 & -0.0000538 & 0.000513 \\
\hline C6 & 0.0004454 & -0.000404 & 0.0400267 & 0.0000224 & -0.0000205 & 0.0011928 \\
\hline D1 & 0.0000047 & -0.000004 & 0.0025892 & 0.0000186 & -0.0000187 & -0.0014627 \\
\hline D2 & 0.0010419 & -0.0015249 & -0.1176412 & 0.0000171 & -0.0000197 & -0.003063 \\
\hline
\end{tabular}




\begin{tabular}{lllllll} 
D3 & 0.0010506 & -0.0007511 & -0.0177963 & 0.0000178 & -0.0000204 & -0.001477 \\
D4 & 0.0004691 & -0.0004882 & 0.0123762 & 0.000017 & -0.0000158 & -0.0009084 \\
D5 & 0.0072367 & -0.0075738 & 0.1321999 & 0.0001089 & -0.0000709 & -0.001653 \\
D6 & 0.0000038 & -0.0000042 & 0.0020211 & 0.000019 & -0.0000218 & 0.0019935 \\
E1 & 0.0000084 & -0.0000079 & 0.0012128 & 0.0000195 & -0.0000191 & -0.0003436 \\
E2 & 0.0013242 & -0.002625 & -0.1247941 & 0.0000429 & -0.0000418 & -0.0008347 \\
E3 & 0.0010188 & -0.0005505 & -0.013439 & 0.0000193 & -0.0000207 & 0.0005212 \\
E4 & 0.0004207 & -0.0003099 & 0.0131795 & 0.0000188 & -0.0000196 & -0.0018299 \\
E5 & 0.0049669 & -0.0050688 & 0.1273632 & 0.0000908 & -0.0000666 & 0.0001761 \\
E6 & 0.0000043 & -0.0000044 & 0.0010742 & 0.0000184 & -0.0000191 & 0.0015754 \\
F1 & 0.0000042 & -0.0000044 & 0.0002018 & 0.0000176 & -0.0000189 & -0.0001177 \\
F2 & 0.0004822 & -0.0015375 & -0.1268739 & 0.0000174 & -0.0000233 & -0.0024814 \\
F3 & 0.0008517 & -0.0004604 & -0.0165925 & 0.0000196 & -0.0000191 & -0.0001067 \\
F4 & 0.0006965 & -0.0002703 & 0.0236493 & 0.0000186 & -0.0000201 & 0.0014059 \\
F5 & 0.004052 & -0.0040384 & 0.1180847 & 0.0000597 & -0.0000483 & -0.0016282 \\
F6 & 0.0000041 & -0.0000042 & 0.0002543 & 0.0000187 & -0.0000202 & 0.0015011 \\
\hline
\end{tabular}


Table 2. The experiment with the gravity generator in the vacuum chamber on June 18, 2020.

\begin{tabular}{|c|c|c|c|c|c|c|c|}
\hline Barometric pressure (mbar) & 1000 & 100 & 10 & 1 & 0.1 & 0.01 & 0.0001 \\
\hline $1^{\text {st }}$ measurement & $-5.69 \mathrm{E}-03$ & $-5.70 \mathrm{E}-03$ & $-5.68 \mathrm{E}-03$ & $-5.69 \mathrm{E}-03$ & $-5.73 \mathrm{E}-03$ & $-5.70 \mathrm{E}-03$ & $-5.68 \mathrm{E}-03$ \\
\hline $3^{\text {rd }}$ measurement & & $-5.72 \mathrm{E}-03$ & $-5.78 \mathrm{E}-03$ & $-5.31 E-03$ & $-5.31 \mathrm{E}-03$ & $-5.38 \mathrm{E}-03$ & $-5.69 \mathrm{E}-03$ \\
\hline $5^{\text {th }}$ measurement & & & & & & $-5.74 \mathrm{E}-03$ & $-5.69 \mathrm{E}-03$ \\
\hline $6^{\text {th }}$ measurement & & & & & & & $-5.27 \mathrm{E}-03$ \\
\hline $7^{\text {th }}$ measurement & & & & & & & $-5.76 \mathrm{E}-03$ \\
\hline $10^{\text {th }}$ measurement & & & & & & & $-5.69 \mathrm{E}-03$ \\
\hline Average (Current) & $-5.73 E-03$ & $-5.70 \mathrm{E}-03$ & $-5.82 \mathrm{E}-03$ & $-5.56 \mathrm{E}-03$ & $-5.58 \mathrm{E}-03$ & $-5.65 \mathrm{E}-03$ & $-5.62 \mathrm{E}-03$ \\
\hline
\end{tabular}

